\title{
The Antisickling Effect of the Arthrospira platensis bilins for Liver Protection: a Modeling, Hypothesis, and Food for Thought
}

\author{
Amro Abd Al Fattah Amara* \\ Protein Research Department, Genetic Engineering and Biotechnology Research Institute, City for Scientific Research and \\ Technological Applications, New Borg Al Arab, Alexandria, Egypt
}

Received: 05 April, 2017; Accepted: 21 June, 2017; Published:18 August, 2017

*Corresponding author: Amro Abd Al Fattah Amara, Protein Research Department, Genetic Engineering and Biotechnology Research Institute, City for Scientific Research and Technological Applications, Alexandria, Universities and Research Center District, New Borg EL-Arab, Egypt, Tel: 203-4593422; Fax: 203 4593497; E-mail: amroamara@hotmail.com; amroamara@web.de

\section{Summary}

The increase in the number of the liver injury and diabetic patients' worldwide and particularly in sub-Sahara in Africa put a signal about that it might be a link between it and the regional diseases such as Malaria and Sickle Cell Anemia (SCA). SCA is a genetic based disease and is one of the liver injury causative agents. Chronic liver injury patients' are susceptible to more complications such as the infection with viruses (e.g. Virus C), diabetic, immune disease, weakness, anemia etc. Arthrospira platensis proves be able to fight and to protect against different diseases at once. It has antiviral, antioxidant, antisickling, nutrient, and edible. In this study their bilins are evaluated for their antisickling effect using molecular modeling aiming to protect patients with SCA particularly the diabetic ones.

In this study molecular modeling for the normal and sickle $\beta$-globin, molecules against five bilins [(1) Red bilin, (2) 21H-Bilin1(22H)-one, (3) 21H-Bilin-1(24H)-one, (4) 1H-Bilin 1 one, and (5) 22H-Biline (21-bilin)] were investigated using protein modeling and docking. MODELLER v 9.8, Hex ver 8.0.0 and Discovery Studio 4.1 Client 4.1.0.14169 (Accelrys software Inc.) were used for modeling and docking. A. platensis bilins are evaluated also by comparing the visualized docking results.

The total energy of the system (the molecule) for all of the used bilins with the sickle $\beta$-globin molecule particularly in both of the presence or the absence of the porphyrin ring prove to improve such energy to be almost equal to that obtained from the normal $\beta$-globin (with or without porphyrin ring). However, porphyrin ring is essential.

A. platensis bilins using molecular modeling prove to be able to stabilize the sickle $\beta$-globin molecules particularly in the presence of the porphyrin ring. As being multifunction, it is recommended either as native biomass or as purified bilins to be used for the treatment of the SCA and the protection against further liver deterioration.

Keywords: Liver injury; Bilin; $\beta$-globin; protein modeling; structure/function/specificity

\section{Introduction}

The proteins structure/function/specificity are governed by their amino acids order, number and arrangement in the protein backbone. Single amino acid change could change the protein property totally. Such change will not effect on the protein family only but will effect on any other macromolecule or micro molecules could gain, or loss affinity to such changed protein. No better example could be described than the SCA. In mammals the $\mathrm{O}_{2}$ binding protein, myoglobin and hemoglobin are among the most extensively early studied proteins. The single amino acid change in the $\beta$-globin (existed on the surface of the protein) cause inefficient $\mathrm{O}_{2}$ transport. The amino acid change lead to a sticky patch on the $\beta$-polypeptide chain of deoxyhaemoglobin, which cause aggregation and precipitation. Such aggregation and precipitation can cause deterioration for the body organs particularly those, which subjected to high blood flow and interaction such as the liver and the spleen. That is not the only type but there are many other variant of hemoglobin mutations. The $\mathrm{Fe}^{2+}$ containing heme group is a highly hydrophobic molecule and requires to be placed in a hydrophobic pocket in hemoglobin quaternary structure. It is important to sign that mutants other than the SCA mutant does not necessarily have to be single amino acid substitutions and not have only to affect the heme pocket [1].

Genetic diseases were well known in the old civilization [2]. The World Health Organization (WHO) (1982) estimated that about five percent of the world populations are carriers of genes for clinically important disorders of hemoglobin [3]. Recently Amara highlight some solutions for avoiding different types of genetic diseases including SCA [4]. Each of the $\beta$ globin genes is represented at least with two copies each in one chromosome gained from the mother and the father to be finally two. So the probability that two incorrect genes find each other will be higher in relatives. Also endemic area with certain incorrect trait or more than one trait of the hemoglobin disease should be 
considered. Prototype mutants must be detected by using DNA sequencing and mapping the possibility of existing of such type of mutant(s) where one or two bases change can lead to a new mutant which not appears yet. Out group marriage will reduce the disease severity and will give more chances to the correct genes to be existed and the mutated genes to disappear [4].

Normal Hemoglobin $\mathrm{Hb} \mathrm{A}$ composition is $\alpha 2 \mathrm{~A} \beta 2 \mathrm{~A}$ with genotype $\alpha \alpha / \alpha \alpha \beta / \beta$. Sickle cell trait hemoglobin $\mathrm{Hb} \mathrm{A}, \mathrm{Hb} S$ its composition $\alpha 2 \mathrm{~A} \beta 2 \mathrm{~A}, \alpha 2 \mathrm{~A} \beta 2 \mathrm{~S}$ with genotype $\alpha \alpha / \alpha \alpha \beta / \beta$ s. Sickle cell disease hemoglobin HbS its composition $\alpha 2 \mathrm{~A} \beta 2 \mathrm{~S}$ with genotype $\alpha \alpha / \alpha \alpha \beta s / \beta$ s. The hemoglobin four chains $2(\alpha) / 2(\beta)$ fitted together to form a globular tetramer with a molecular weight of approximate 64000 , a structure that for $\mathrm{Hb} \mathrm{A}$, is abbreviated as $\alpha 2 \beta 2$. The two kinds of chains are almost equal in length, the $\alpha$ chain having 141 amino acids and the $\beta$ chain $146 . \alpha$ and $\beta$ chains are encoded by genes at separate loci (the $\alpha$ locus on chromosome 16 and the $\beta$ locus on chromosome 11). In addition to $\mathrm{Hb} \mathrm{A}$, there are five other normal human hemoglobin's, each of which has a tetrameric structure comparable to that of $\mathrm{Hb} \mathrm{A}$ in consisting of two $\alpha$ or $\alpha$-like chains and two non- $\alpha$ chains [5].

Liver problems are reported in patients with SCA in 37\% cases [6]. Those patients have abdominally meteorism, right upper quadrant pain, or acute painful hepatomegaly. In general different form of liver injury in most cases as cholestatic. Liver infarction has been reported in $34 \%$ of autopsies [7]. The SCA associated blood viscosity predisposes to infarction $[8,9]$. The liver enzymes activity increased abnormally $[10,11]$. The causing agent in case of SCA patients thought to be obstruction of sinusoidal flow of masses of sickled erythrocytes, trapping them in the liver [12].

There is an increasing in the number of publications, which introduce A. platensis as a proposed candidate could be used to reduce the antisickling agent.

This study investigates $A$. platensis bilins structures, which might be able to reduce the sickling process as well as to show that bioinformatics tools must give more concern to the DNA sequences. A. platensis bilins have proved to have many useful activities especially as antiviral, antitumor, antioxidant and antisickling. The heme and hemoglobin proteins were docked against five bilins from A. platensis. This study refreshes the scientific aim to control and to treat such illness happened by a one nucleotide change. And can be only avoided by avoiding the marriage from the same group. Encourage the marriage from out group is the correct and the simplest solution. And also to protect from further deterioration in organs such as the liver, where diabetic SCA patients will subject to sever liver injury.

\section{Material and Methods}

\section{Bilins}

The three-diminution structures for five $A$. platensis bilins were used in this study to investigate their abilities to dock with sickle hemoglobin and $\beta$ - globin protein models. The chemical formula and name of the used bilins are:
1. Red bilin [Also known as: CPD-7063, (7S,8S, 101R)-8-(2carboxyethyl)-17-ethyl-19-formyl-101-(methoxycarbonyl)3,7,13,18-tetramethyl-2-vinyl-8,23-dihydro-7 H-10,12ethanobiladiene-ab-1,102(21H)-dione]. Which have molecular formula: C35H38N407-2; molecular weight: $626.69882 \mathrm{~g} / \mathrm{mol}$; InChI Key: HMDDKKOMBDRDIA-DSJLEYPNSA-L. Its IUPAC name is: $\quad[3-[(2 Z, 3 S, 4 S, 5 Z)-5-[(4-e t h e n y l-3-m e t h y l-5-o x o p y r r o l-2-y l)$ methylidene]-2-[2-[(3-ethyl-5-formyl-4-methyl-1H-pyrrol-2-yl) methyl]-5-methoxycarbonyl-3-methyl-4-oxido-2,3-dihydro1H-cyclopenta[b]pyrrol-6-ylidene]-4-methylpyrrolidin-3-yl] propanoate].

2. 2.2 1H-Bilin-1(22H)-one,2,3,7,8,12,13,17,18-octaethyl-19methoxy-, 113435-10-2, MolecularFormula: C36H48N402, Molecular Weight: $568.79192 \mathrm{~g} / \mathrm{mol}$, InChI Key: QCYVKTHGTVWFSV-UHFFFAOYSA-N. Its IUPAC name is: [5-[[5-[[5-[(3,4-diethyl-5-methoxypyrrol-2-ylidene)methyl]3,4-diethyl-1H-pyrrol-2-yl]methylidene]-3,4-diethylpyrrol-2ylidene]methyl]-3,4-diethylpyrrol-2-one].

3.AGN-PC-002GJ1,21 H-Bilin-1 (24 H)-one,19-hydroxy-, $21 \mathrm{H}$-Biline-1,19-dione, 2 2,24-dihydro-,142550-15-0, 58828-89-0, Molecular Formula: C19H14N402, Molecular Weight: $330.34006 \mathrm{~g} / \mathrm{mol}$, InChI Key: MQHWQQCOXHUNCSUHFFFAOYSA-N, Its IUPAC name is: 5-[[5-[[5-[(5-oxopyrrol-2ylidene)methyl]-1H-pyrrol-2-yl]methylidene]pyrrol-2-ylidene] methyl]pyrrol-2-one.]

4. 1H-Bilin-1-one [Also known as: AGN-PC-00FTAO, 66560-676]. Which have molecular formula: $\mathrm{C} 19 \mathrm{H} 12 \mathrm{~N} 40$; molecular weight: 312.32478 g/mol; InChI Key: VGJBOZZPXZVBBI-UHFFFAOYSA-N. Its IUPAC name is: 5-[[5-[[5-(pyrrol-2-ylidenemethyl)pyrrol-2ylidene]methyl]pyrrol-2-ylidene]methyl]pyrrol-2-one

5. 22H-Biline, 21H-Bilin, 22H-Bilin, AC1OAGP5, SureCN139406, AGN-PC-02LS4D

Molecular Formula: C19H14N4, Molecular Weight: 298.34126 g/mol, InChI Key: PPRBOEHFGAHFGC-UHFFFAOYSA-N. Its IUPAC name is: 2-(pyrrol-2-ylidenemethyl)-5-[[5-(pyrrol-2ylidenemethyl)-1H-pyrrol-2yl] methylidene] pyrrole

Another bilins are existed but will not included in this study

The five bilin molecules were downloaded from PubChem (www.ncbi.nlm.nih.gov/pccompound) and saved as SDF format files [13]. The chemical structure of the molecules is given in (Table 1).

\section{The $\beta$-globin sequences and software used in this study}

The amino acids sequences of both normal and sickle $\beta$-globin are represented by the following amino acid sequences.

\section{A: The normal $\beta$-globin}

VHLTPEEKSAVTALWGKVNVDEVGGEALGRLLVVYPWT QR F F ESF G D L S T P D A V M G N P KV KA H G KKV L G A F S DGLAHLDNLKGTFATLSELHCDKLHVDPENFRLLGNVLVCVLAHHFGKEFTPPVQAAYQKVVAGVANALAHKYH 


\section{B: The Sickle $\beta$-globin}

VHLTPVEKSAVTALWGKVNVDEVGGEALGRLLVVYPW T QR F F E S F G D L S T P D A V M G N P KV KA H G K KV L G AF S DGLAHLDNLKGTFATLSELHCDKLHVDPENFRLLGNVLVCVLAHHFGKEFTPPVQAAYQKVVAGVANALAHKYH

Normal and sickle $\beta$-globin amino acids sequences were the start point of this study. The sequence was obtained from the www.ncbi.nlm.nih.gov (Blast.ncbi.nlm.nih.gov/Blast.cgi) [14].

\section{The software used in this study}

\section{Software for Modeling}

One published sickle hemoglobin model was used [15] The $\beta$-globin protein model was generated using the software MODELLER v 9.8 [16].

\section{Software for docking}

"Hex" is a Molecular Graphic Program (Hex's Home Page: http://www.loria.fr/ ritchied/hex/) for calculating and displaying feasible docking modes of pairs of protein and DNA molecules $[17,18,19]$. Hex software can also calculate ProteinLigand Docking, assuming the ligand is rigid, and it can superpose pairs of molecules using only knowledge of their 3D shapes [20]. It uses Spherical Polar Fourier (SPF) correlations to accelerate the calculations and its one of the few docking programs which has built in graphics to view the result [18]. Simply, the protein pdb is loaded from the "File $>$ open $>$ receptor" and the bilin or the porphyrin ring loaded from "File $>$ open $>$ ligand" and then from the control option docking is selected and the parameter in is used (Figure 1). The binding energy result is normally negative, stating that a better binding affinity is established from the highest negative result. Low (negative) energy indicates a stable system.

To determine the behavior of both of the protein molecules under study and whether we need high negative energy or lower ones; porphyrin ring has been docked firstly against both of the normal and the sickle $\beta$-globin molecules which obtained from the Modeller software and the published sickle hemoglobin model as above.

The five used bilins and one porphyrin ring which used in this study are summarized in (Table 2). The docked molecules' 3D structures have been saved as pdb files and visualized in to show the different interactions (Table 3,4).

\section{Software for the molecules study}

The software Discovery Studio 4.1. Client 4.1.0.14169 (Accelrys software Inc.) was used to visualize the docking of the bilins with the proteins models and to show ligands binding sites and the other analysis for the docked molecules [21].

For better 3D structure, the background of the images have been converted to white and the 3D image have been adjusted and saved. All of the docking images have been putted in tables to enable better comparisons between the interaction between the $\beta$-globin and the bilins (Table 3,4).

\begin{tabular}{|c|c|c|c|c|c|c|c|c|c|c|}
\hline \multirow{2}{*}{$\begin{array}{l}\text { Molecules } \\
\text { name }\end{array}$} & \multicolumn{4}{|c|}{ Etotal (total energy of the system) ${ }^{*}$} & \multicolumn{6}{|c|}{ Differences } \\
\hline & $\begin{array}{l}\text { Normal } \\
\beta \text {-globin } \\
\text { (without } \\
\text { porphyrin } \\
\text { ring) }\end{array}$ & $\begin{array}{l}\text { Normal } \\
\beta \text {-globin } \\
\text { (with } \\
\text { porphyrin } \\
\text { ring) }\end{array}$ & $\begin{array}{l}\text { Sickle } \\
\beta \text {-globin } \\
\text { (without } \\
\text { porphyrin } \\
\text { ring) }\end{array}$ & $\begin{array}{l}\text { Sickle } \\
\beta \text {-globin (with } \\
\text { porphyrin } \\
\text { ring) }\end{array}$ & $\begin{array}{l}\text { between } \\
\text { column } 1 \\
\text { and } 2\end{array}$ & $\begin{array}{l}\text { between } \\
\text { column } 1 \\
\text { and } 3\end{array}$ & $\begin{array}{l}\text { between } \\
\text { column } 1 \\
\text { and } 4\end{array}$ & $\begin{array}{l}\text { between } \\
\text { column } \\
2 \text { and } 3\end{array}$ & $\begin{array}{l}\text { between } \\
\text { column } 2 \\
\text { and } 4\end{array}$ & $\begin{array}{l}\text { between } \\
\text { column } 3 \\
\text { and } 4\end{array}$ \\
\hline Red bilin & -380 & -322.5 & -318.2 & -305 & -57.5 & -61.8 & -75 & -4.3 & -17.5 & -13.2 \\
\hline $\begin{array}{l}\text { 21H-Bilin- } \\
\text { 1(22H)-one }\end{array}$ & -352.1 & -162 & -163.9 & -149 & -190.1 & -188.2 & -203.1 & 1.9 & -13 & -14.9 \\
\hline $\begin{array}{l}\text { 21H-Bilin- } \\
\text { 1(24H)-one }\end{array}$ & -279 & -122 & -111 & -123.1 & -157 & -168 & -155.9 & -11 & 1.1 & 12.1 \\
\hline $\begin{array}{l}\text { 1H-Bilin } 1 \text { one, } \\
\text { and }\end{array}$ & -277.7 & -137.1 & -140 & -131.4 & -140.6 & -137.7 & -146.3 & 2.9 & -5.7 & -8.6 \\
\hline $\begin{array}{l}\text { 22H-Biline(21- } \\
\text { bilin) }\end{array}$ & -349 & -164.1 & -161.2 & -159.4 & -184.9 & -187.8 & -189.6 & -2.9 & -4.7 & -1.8 \\
\hline
\end{tabular}

*The EShape (energy of shape only approach) is same as the ETotal.

$\$$ the ETotal and EShape of the normal and sickle $\beta$-globin with porphyrin ring are -821 and -516 respectively 


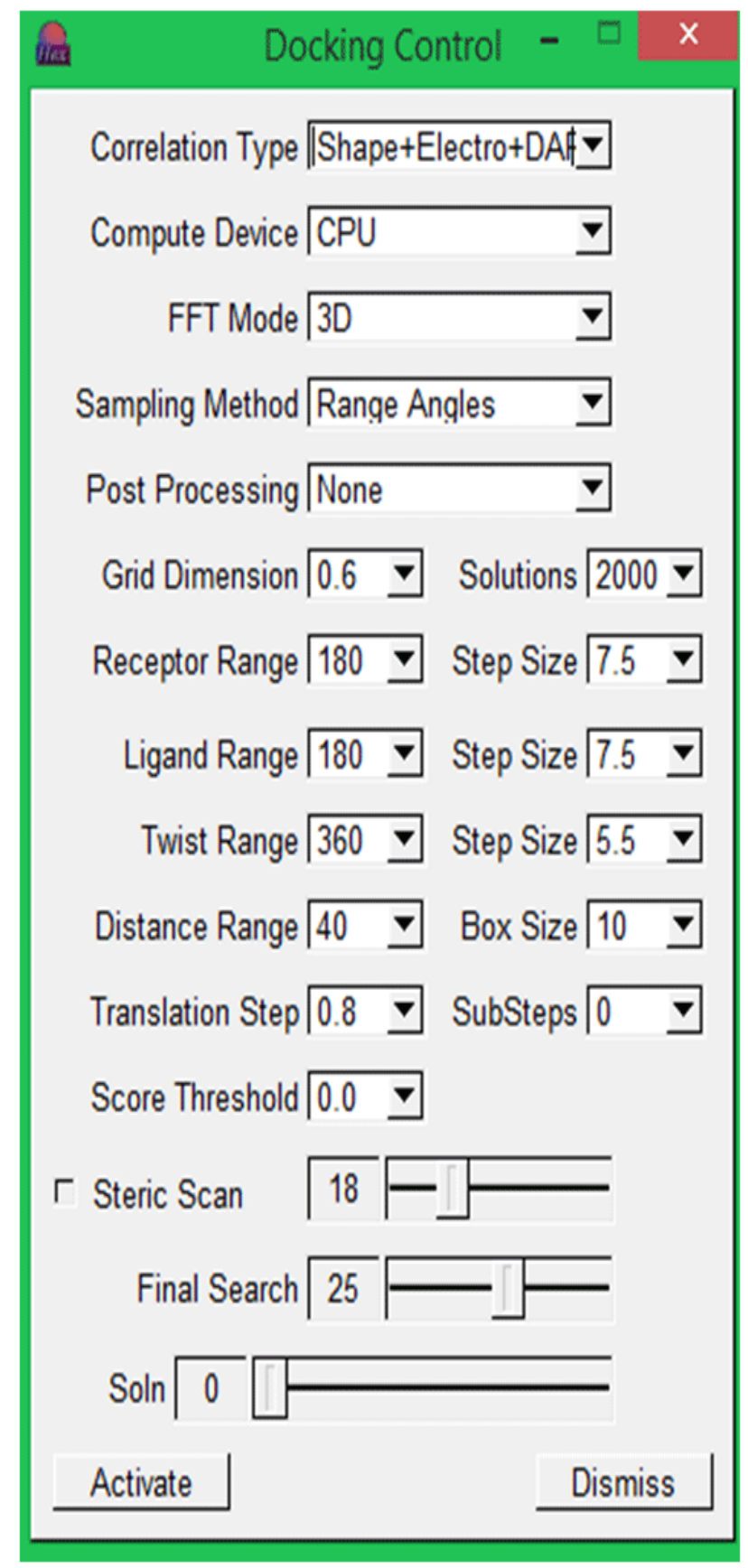

Figure 1: The Hex software docking parameters

\section{Results and Discussion}

Our genes are the codes for the proteins in our bodies. Understanding our genes and our proteins will help us to avoid different illness and to design new drugs. Such drugs can be so simple and can be supplied as natural products in the form of food or edible plants. Such natural products can provide us with what the defected genes could not do. Vitamin C is the most famous example. Others forms might can improve certain function like the structure of the SCA defect protein. We are in need to do complicated research to find solutions for some degenerative diseases which alter our macromolecules structure hence their functions and specificity. However, avoiding such type of diseases is so simple. It is just by avoiding the marriage from the same group and from those which have the same disease trite (should not marry from each other). SCA is a known genetic disease in West sub-Sahara in Africa, in the Mediterranean region and other places worldwide [4].

SCA which also named as hemoglobin S disease or hemoglobin SS diseases. After losing the oxygen SCA cells soon stimulate holly leaves or their crescents. Soon they become filamentous and spirculated. Single gene of hemoglobin $S$ and the other is A named sickle cell trait. Individuals have hemoglobin $S$ and $\beta$ - thalassemea ( $\beta$-thal) are both prevalent (Greeks and Italians) having a high incidence of S-thalssemia (S- $\beta$-thalassemia) [22].

Additionally, scientists, particularly, those from the SCA endemic regions and countries, have summarized their experiences as well the experience gained from their communities in controlling the disease side effect.

Smith and Wood in their book about the Biological Molecules (1991) have written: 'The present-day distribution of effective hemoglobin's has arisen from the accumulation of harmless mutations, early death of individuals with harmful mutation, this confers a selective survival advantage such as increased resistance to malaria, as is the case which sickle cell disease' [1].

For that, lethal mutant are unable the transform their genotype to the second generation. While mutant which gives the minimum survives until the appearance of the seconded generation will do and will be transferred from generation to generation. However there is a $50 \%$ chance that the correct chromosome transfers instead of the one which has the mutant. Alternatively, existing of two globin mutant (on $\alpha$ and $\beta$ which existed in two chromosomes) will increase the chance of the transfer of globin disease.

Hemoglobin is the oxygen carrier tetrameric molecule and can be found in vertebrate red blood cells, in some invertebrates and in the root nodules of legumes [23]. Each subunit is composed of a polypeptide chain, globin, and a prosthetic group, heme, which is an iron-containing pigment that combine with oxygen and gives the molecules its oxygen-transporting ability. SCA is a global disease and for the Mediterranean and the Africans communities is a local disease [24]. Livingstone, has described in detailed the roles which affect the percentage and the distribution of the SCA in West Africa. From the time of specifying the role of the heredity (the most critical one) till producing artificial blood and artificial oxygen carrier, the scientific progress and the scientist effort did not stop [25].

The biological system is sensitive for the chemical structure. Enzymes could be so specifics. Other protein forms could be also be very sensitive. Red Blood Cells (RBCs) could differentiate between $\mathrm{O}_{2}$ and $\mathrm{CO}_{2}$. The conditions and the structure draw the 
Table 2: Six macromolecules and six molecules (five bilins and one porphyrin ring) used in this study

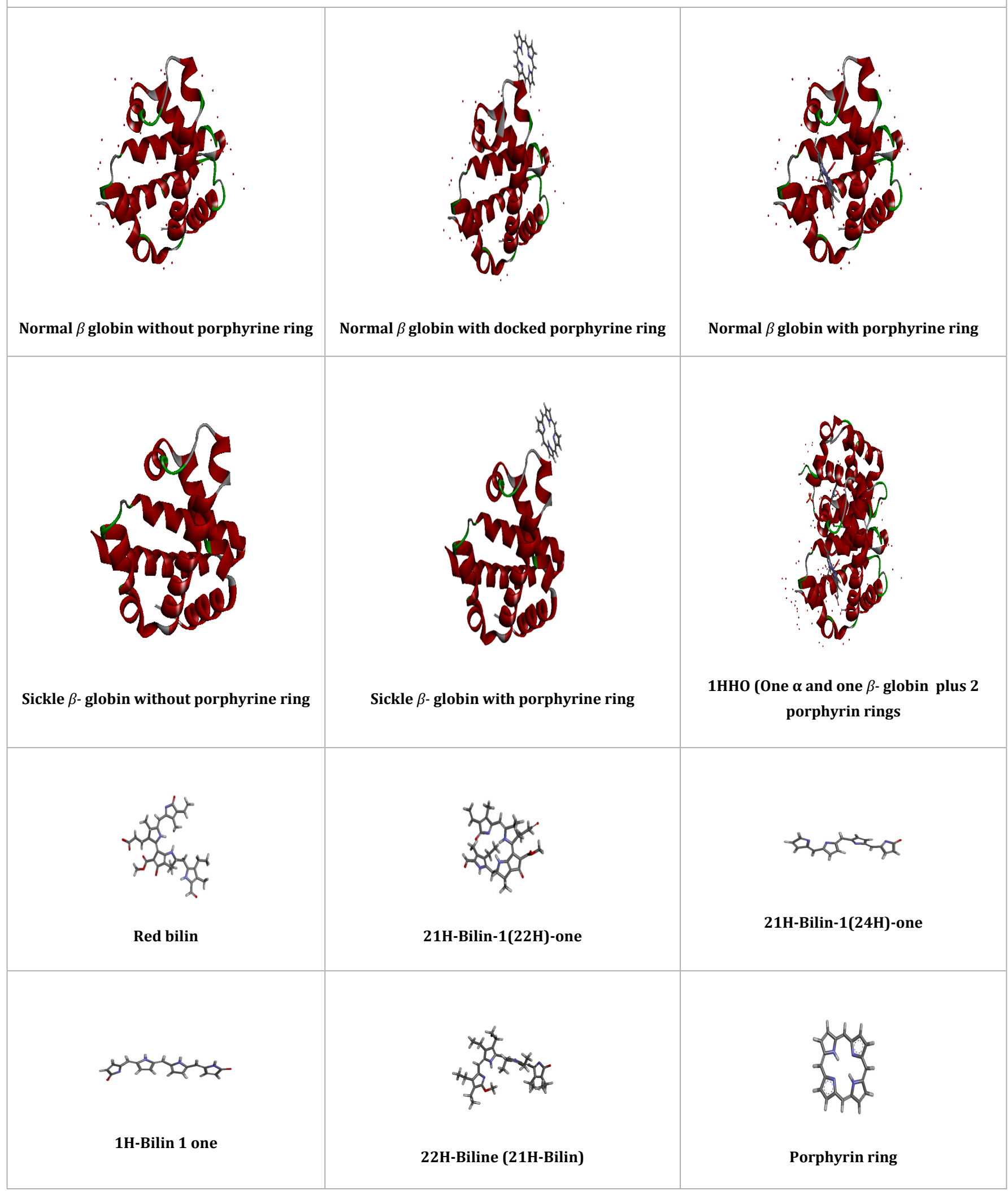


Table 3: The different docking results between the five $\beta$-globin macromolecules and the five bilins

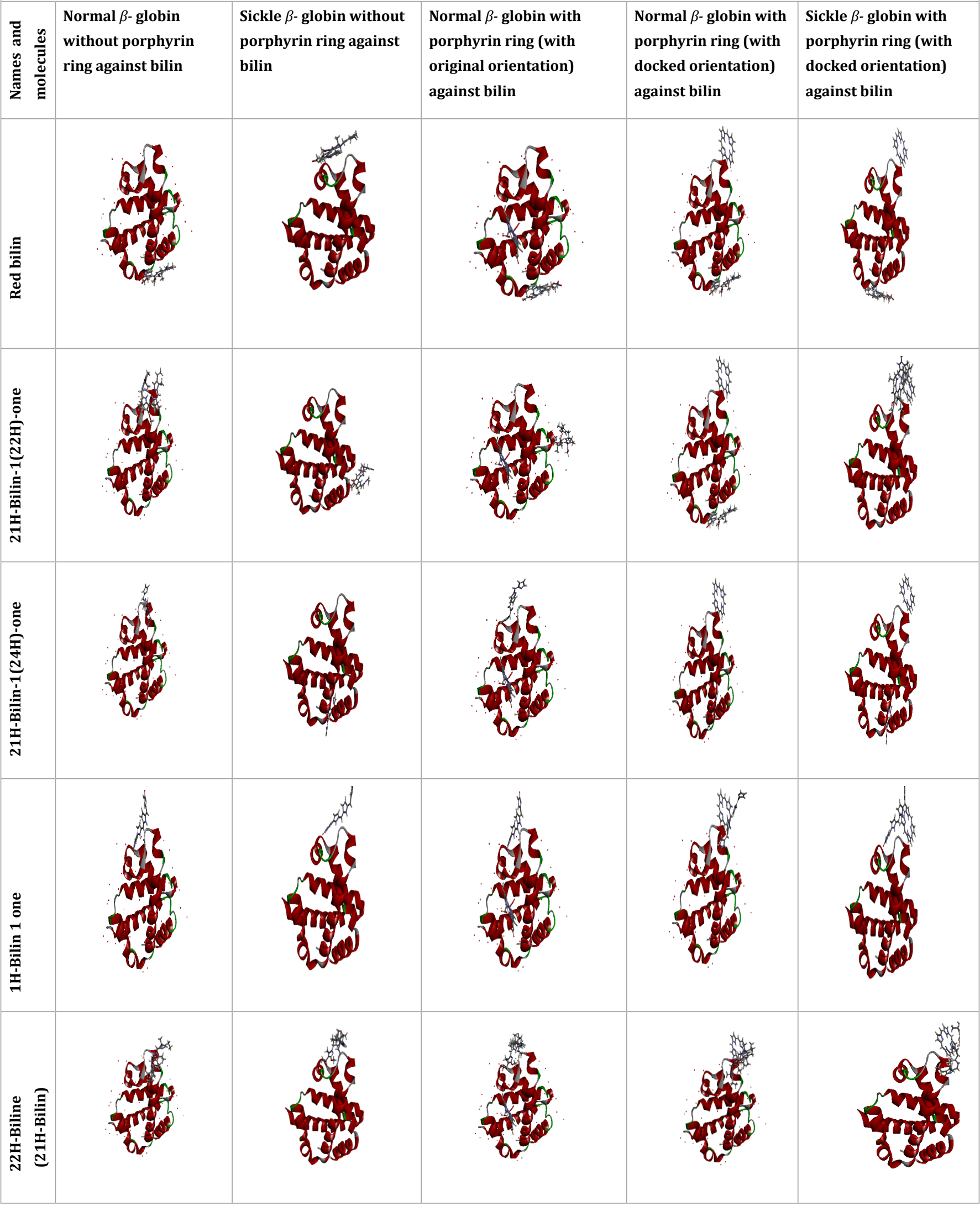

Citation: Amro Abd Al Fattah Amara (2017) The Antisickling Effect of the Arthrospira platensis bilins for Liver Protection: a Page 6 of 12 Modeling, Hypothesis, and Food for Thought. SOJ Biochem 3(1):1-12. DOI: http://dx.doi.org/10.15226/2376-4589/3/1/00123 
Table 4: Different molecules surface interaction with the $\beta$-globin macromolecules

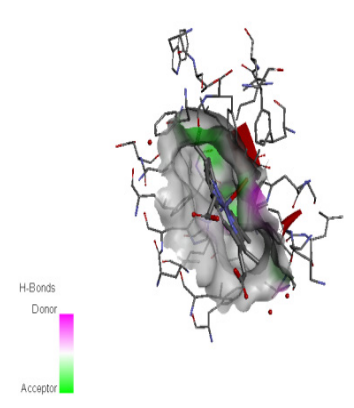

Normal porphyrin ring (surface original)

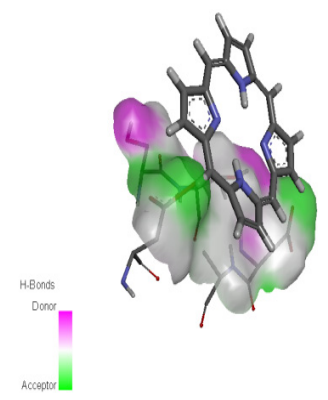

Sickle $\beta$-globin against porphyrin ring

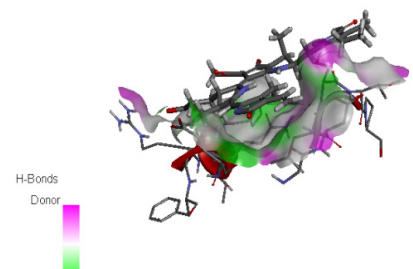

Red bilin Sikle $\beta$-globin (surface original)

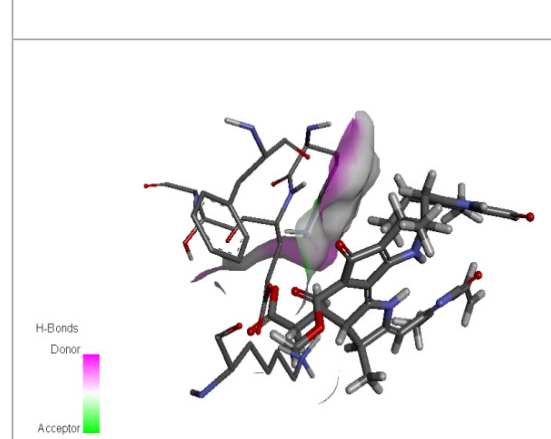

21H-Bilin-1(22H)-one sickle $\beta$-globin (surface original)

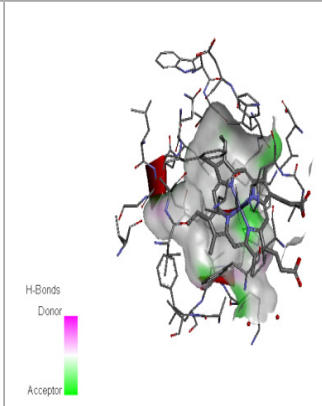

Normal porphyrin ring (surface original)

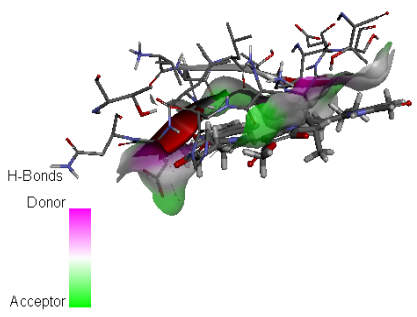

Red bilin against normal $\beta$-globin alone (surface original)

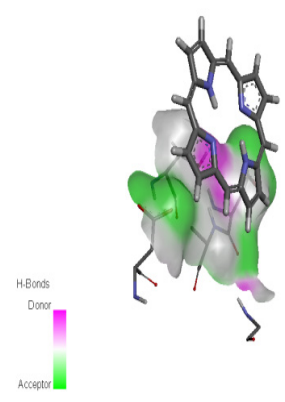

$\beta$-globin against docked porp

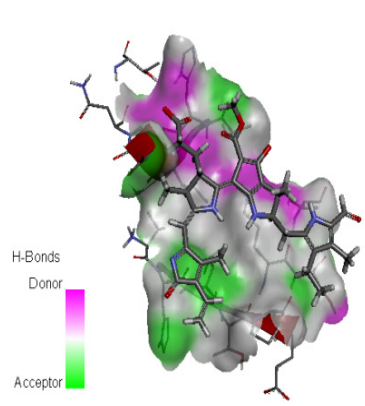

Red bilin against normal $\beta$-globin alone (surface modified)

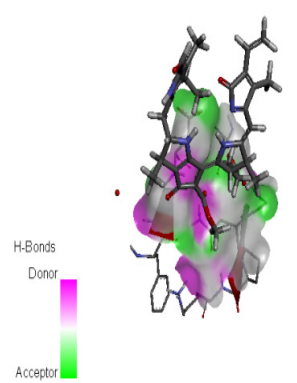

21H-Bilin-1(22H)-one against normal $\beta$-globin alone (surface original)

21H-Bilin-1(22H)-one sickle $\beta$-globin (surface modified)
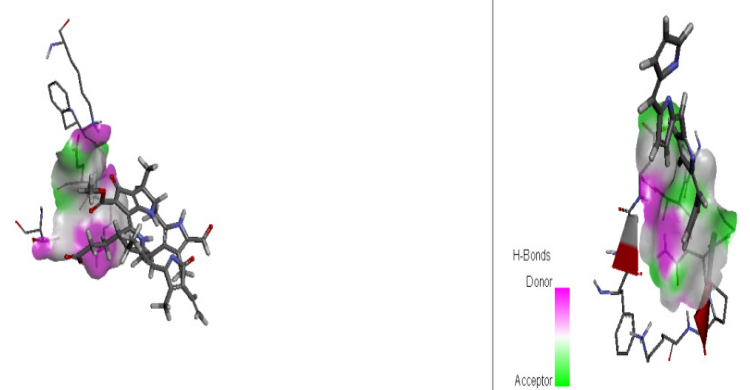

21H-Bilin-1(24H)-one against normal $\beta$-globin alone (surface original) 


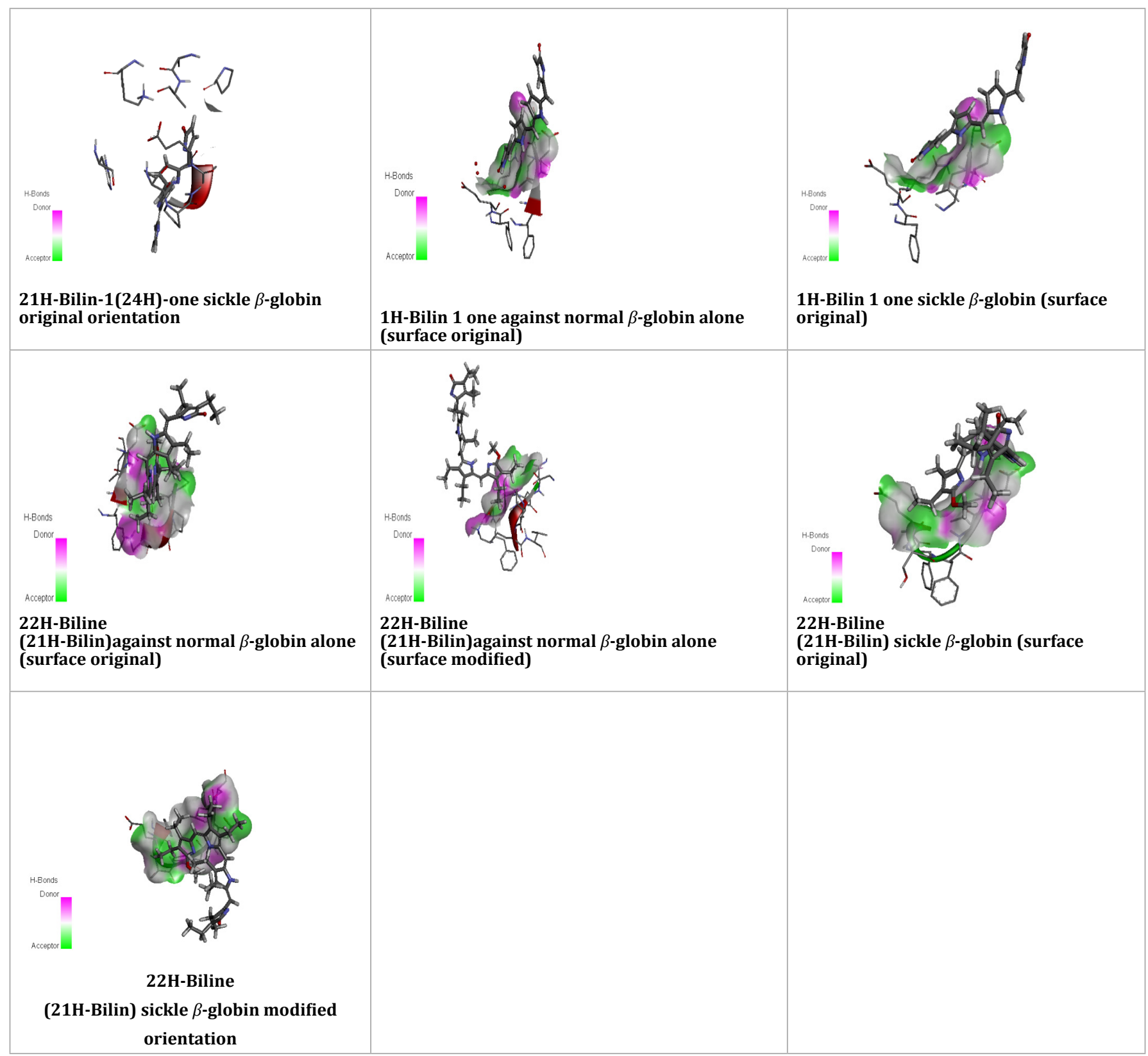

function of the RBCs; where they gain $\mathrm{O}_{2}$ and where they lose it; where they gain $\mathrm{CO}_{2}$ and where they lose it. Such specificity is not only in the heme active amino acids but also in the atoms of $\mathrm{O}_{2}$ and $\mathrm{CO}_{2}$. For that it is important to investigate molecules could be able to stabilize the sickle hemoglobin particularly during the $\mathrm{CO}_{2}$ stage which is the aim of this study.

The HBB gene provides instructions for making $\beta$-globin. Various versions of $\beta$-globin result from different mutations in the HBB gene. One HBB gene mutation produces an abnormal version of $\beta$-globin known as Hemoglobin S (HbS). Other mutations in the HBB gene lead to additional abnormal versions of $\beta$-globin such as Hemoglobin $\mathrm{C}(\mathrm{HbC})$ and Hemoglobin E (HbE). HBB gene mutations can also result in an unusually low level of $\beta$-globin; this abnormality is called $\beta$-thalassemia. When oxygen is removed from sickle hemoglobin, those molecules change their shape and combine with one another. The Red blood cell structure changes from ring to sickle shape in the absence of the oxygen. This causes blood to clots and deprives vital organs from their supply of blood, resulting in pain, intermittent illness, and in many cases, a shortened life span. The only difference between normal and sickle cell hemoglobin is that in each $\beta$ - chain, one glutamic acid is replaced by one valine. Valine, unlike glutamic acid, contains a nonpolar group. The result is a hydrophobic "sticky" region that can interact with hydrophobic region on neighboring molecules, producing the observed clumping. A slight change in the $\beta$-globin 3D structure will induce a change in the configuration of it when it interacts with its neighboring subunits of the hemoglobin.

The macromolecules are very sensitive to any effect could 
effect on their structures/functions. Such changes can be classified based on different strategies. But, classifying them regarding to the type of the effective changes will lead to a more focusing on the positive and negative factors and the possible solutions for illness problems. For that, classifying the factors effect on the macromolecules structure/function can be divided to the following groups and probability:

1. Wild type macromolecules in correct environment that will give correct function(s).

2. Wild type macromolecules in optimized environment better than the standard that will optimize the function(s) but can cause deterioration for the macromolecules hence illness.

3. Wild type macromolecules in condition less than the standard this will give less functions and could lead to the deterioration for the macromolecules.

4. Wild type macromolecules in incorrect environment that will give a temporary incorrect function but they are still able to do correct function in the suitable conditions.

5. Mutant macromolecules in environment let them work like wild type that will cause deterioration while the macromolecules do an extra job and in normal case they do less activity.

6. Mutants in environment same like the best condition for the wild type. This might not give the same result of the wild type or protect the macromolecules from further deterioration.

7. Incorrect macromolecules find some support from other macro or micro molecules to do their job with less effort or to fit or repair some of the existed problems due to the change in their structures hence their physicochemical properties. This group is most one fit with the aim of this study.

\section{From the simple classification of the macromolecules as above it can be concluded that:}

1. Wild type macromolecules even correct but should be maintained in the conditions which did not turn them to mutants as in case of the DNA or the RNA and not deteriorate them such as in case of the proteins and short peptides.

2. Mutated macromolecules should be lead to illness in most cases where the probability of their deterioration is higher than the wild type because they are doing incorrect function or do more effort to reach the wild type functions and activity.

3. Both the macromolecules' situation either being wild or mutant as well as the surrounding environment plays a significant role in their functions.

4. Sick macromolecules should be treated as sick and their work should not be optimized while that will lead to further deterioration. Perhaps a good example here could be given about the drugs which prevent or decrease the pain. In fact most of them stop our failing towered the pain target but the damage is continuous and not stop. It is better to lessen well for the pain signals in our bodies. Given our bodies enough rest and enough time to recover is far better than force it to work incorrectly in the presence of pain. Pain is the best friend for observing problems in our bodies.

5. Factors which could improve the function of the mutant macromolecules without putting load on the targeted macromolecule itself will be the best solution. It is like the competition between the oxidants plus the free radicals against our endogenous antioxidant where high oxidants and free radicals will deteriorate our endogenous antioxidants particularly if there is an existing continuous source for them either by bypassing it, exhaust the cells, damage the DNA and the RNA including those responsible for the antioxidant system. Supplying the body with suitable exogenous antioxidant will overcome all of such problems. However, continuous supplying with exogenous source will deteriorate the endogenous antioxidant too!

6. The type of the changes must be clear for better treatment. Is it permanent such as mutants? Or only due to environmental and conditional changes but the macromolecules still correct?

7. One should not put his macromolecules in the optimum conditions where optimum conditions is in the top of the peak and will lead soon to drops and vice versa will give the same result.

8. Balanced life is required.

SCA might be more interested as a subject for the Mediterranean area and the sub-Saharan West region in Africa. Back to the first observation where the Flamingo birds are interested to feed themselves by A. platensis(Spirulinaplatensis) in Chad to be able to follow their long migration trips. Flamingo birds which are appeared healthy and reddish is a signal that such an African cyanobacteria was the early signal for the local individuals that such edible cyanobacteria can be eat as food $[26,27]$.

After generating the sickle $\beta$-globin and docking the porphyrin ring with both of the normal and sickle $\beta$-globin five molecules were existed as pdb files. They are:

1. Normal $\beta$-globin without porphyrin ring.

2. Normal $\beta$-globin with porphyrin ring in its native form.

3. Normal $\beta$-globin with docked porphyrin ring.

4 . Sickle $\beta$-globin without porphyrin ring.

5. Sickle $\beta$-globin with docked porphyrin ring.

The five molecules each has been docked against each of the A. platensis bilins.

The determined normal and the sickle $\beta$-globin molecules (which obtained from the Modeller software and the published sickle hemoglobin model as above) were ETotal (the total energy of the system) "-821.0" for the normal $\beta$-globin and -516.9 for the sickle $\beta$-globin. The Etotal prove that the interaction between the porphyrin ring and the normal $\beta$-globin are more negative hence, 
more stable interaction is existed.

Etotal in case of the normal $\beta$-globin with all of the five bilin show less energy than that in case of the presence of porphyrin ring (without $\mathrm{Fe}^{2+}$ ) which means more stable interactions. That means the existence of correct interaction between the bilins and the normal $\beta$-globin. In case of sickle cell $\beta$-globin the same result was obtained in all cases. That proves that the presence of the porphyrin ring reduces the total energy of the system, which is logic where the existence of two molecules interacting with a single protein probably will lead to reduce the interaction force by each molecule if compared with the force determined if the each molecule interact in individual case.

Normal $\beta$-globin only (without porphyrin ring) and with bilin show in all cases less total energy than that of sickle $\beta$-globin , which prove that sickle $\beta$-globin molecule with the bilins have good interaction.

However, in presence of porphyrin ring in case of sickle $\beta$-globin in case of Red bilin, 21H-Bilin-1(22H)-one, 1H-Bilin 1 one, and 22H-Biline(21-bilin) show increase in the system total energy than that in case of the absence of porphyrin ring.

Only the presence $21 \mathrm{H}$-Bilin-1(24H)-one show decrease in the total system energy in case of the presence of porphyrin ring. So, $21 \mathrm{H}$-Bilin-1(24H)-one is a molecule that can stabilize the $\beta$-globin.

However, in general, the presence of the porphyrin ring or a bilin or both increases the total energy of the system except in $21 \mathrm{H}$-Bilin-1(24H)-one with sickle $\beta$-globin. Small increase shown also between $21 \mathrm{H}$-Bilin-1(22H)-one with normal $\beta$-globin and with porphyrin ring if compared with $21 \mathrm{H}$-Bilin-1(22H)-one with sickle $\beta$-globin (without porphyrin ring).

The slight decrease in the negativity in case of Red bilin, $21 \mathrm{H}$-Bilin-1(22H)-one, $1 \mathrm{H}$-Bilin 1 one, and 22H-Biline(21-bilin) as in show that such molecules still so close to the negativity of the obtained from the normal $\beta$-globin (Table 1). The five bilin successfully readjust the Etotal of the molecules in case of the presence of porphyrin ring and the differences in the system in case of column 2 and 4 is negligible in all cases and range from “-17.5” in case of Red bilin to "+1" in case of $21 \mathrm{H}-$ Bilin- $1(24 \mathrm{H})$ one.

In conclusion the variation between normal $\beta$-globin and sickle ones are significant (in case of absence of porphyrin ring) as in column 1 and 2 but not significant in case of column 2 and 4 which prove that porphyrin ring is essential in the $\beta$-globin structure and that bilins could play a significant role in establishing the $\beta$-globin molecules.

One should remark that Hex program is a windows docking software, however, it can give preliminary judgment for the differences and the possibilities of using bilins to treat sickle $\beta$-globin. The used CPU is "Hp Compaq nc 4200". In addition, the used Hex software is Hex ver. 8.0.0. However, it is a remark that slight variation in the molecule can be sound in the total energy of the system (the molecules or the molecules and its legends). In addition, the use of the same condition and the same computer will reduce any variation due to different systems' error. The obtained results have enough variation to prove that the fiveused bilins could stabilize the sickle $\beta$-globin particularly in the presence of the porphyrin ring, and that $21 \mathrm{H}$-Bilin- $1(24 \mathrm{H})$-one might be the best tested molecules.

The study is also highlight the importance of the porphyrin ring in the controlling of the sickle cell anemia side effect.

Bilins which are derived from the edible cyanobacteria the $A$. platensis and which reported in quite enough studies [data not shown] to have antisickling effect, could play a significant role in reducing the side effect of the SCA. "Target the target which targets you", words might be a key for controlling the SCA and other degenerative disease, where many of such diseases is treated from their end point while, their starting point located in avoiding the marriage from the same group as described in details by Amara [4].

A. platensis might be the ideal edible food for both of the protection and the treatment, in case of patients with both of SCA and diabetic. It is well known for its antioxidant activity, gamma fatty acid, antiviral, and antisickling. For that, it can react with a different target at once to protect against any of the exponential deterioration could effect on the other organs. Hex software is recommended for being used to evaluate more molecules to find the ideal solution for the SCA.

One should put in his account that this study represents models conducted in computer study and, to validate any of the obtained result; in vivo experiment should be conducted (in future study).

The purpose of preparing each of the normal and sickle $\beta$-globin using MODELLER software is to reduce any differences from using an original x-ray based structure obtained from the web. However, a published model was used for such purpose. Additionally, porphyrin molecule without $\mathrm{Fe}^{+2}$ was used. All those factors should be considered during conducting study similar to this study. Docking of the normal and sickle $\beta$-globin against prophyrin ring was for the purpose to find the direction of the molecules' stabilization in case of the $\beta$-globin molecule. Yes, the more negativity means more stability, but in our case stability might not be required but equality is the correct target. So stable molecular interaction in this study case might not enable correct oxygen exchange. But equality with the native normal $\beta$-globin is the correct key for investigation.

In our case the docking of the normal $\beta$-globin with porphyrin ring give total system energy equal to -821 while the docking of the sickle $\beta$-globin against the porphyrin ring give -516.9 . Sickle $\beta$-globin is clearly less stable in its interaction with the $\beta$-globin; considering that the data obtained from the interaction between the normal $\beta$-globin and the porphyrin ring is the ideal one 
$(-821)$. That might explain the source of the deterioration in case of presence of SCA disease. The purpose might not in the structure only but in the energy as well.

Not all of the pdb files obtained in this study are represented. However, the included images explain also for some extent that it might be used to prove or disprove some facts. Images might be describe in better way where the molecules are interacts and which place are preferable on the protein backbone (to be stabilize or destabilize it). Such docking might not represent the absolute fact, but it will give somehow preliminary judgment and an overall view about what could be happened if such molecules are present in our bodies. Such molecules are derived from edible source so they might be safe if used in an adequate amount. A fact; even seem to be correct in case of using the A. platensis native biomass but, need to be proved in case of purified compounds.

For the five bilins it is clear from their models as in that the bilin have good interaction with both of the $\beta$-globin molecules (Normal and sickle) (Table 3,4). Apparently, the smaller the molecules the better it become inside the 3D structure. The lager the molecule it tends to attach on the surface of the $\beta$-globin molecules. The existence of porphyrin ring or their absence shows different-results. That proves the sensitivity of the process and the importance of the porphyrin ring in the structure of the $\beta$-globin, the hemoglobin and the RBCs overall structure.

Based on the five bilin docking data with the $\beta$-globin; the docking process is very sensitive.

1. In case of Red bilin the bilin bind to the $\beta$-globin in different cases nearly in the same site, except in case of sickle $\beta$-globin without porphyrin ring.

2. $21 \mathrm{H}$-Bilin-1(22H)-one show different binding property for all of the five molecules. $21 \mathrm{H}$-Bilin-1(22H)-one is sensitive to any change in the $\beta$-globin different molecules.

3. $21 \mathrm{H}$-Bilin-1(24H)-one show different binding sites between normal and sickle molecules but nearly the same in either normal or to the sickle molecules.

4. H1-Bilin-one is bind nearly to the same in place in all of the five macromolecules and show competition against the porphyrin ring.

5. $22 \mathrm{H}$-Bilin $(21 \mathrm{H}$ Bilin) bind in the same place from the five macromolecules and show competition against the porphyrin ring.

Supposing that $\beta$-globin with normal porphyrin ring which obtained without modification from the 1 HHO model with native porphyrin ring orientation (no docking) is the most correct macromolecules, in such case Red bilin, 1H-Bilin 1 one and $22 \mathrm{H}$-Bilin $(21 \mathrm{H}$ Bilin) will be the best molecules which expected to support the normal and the sickle $\beta$-globin without the interferes with the porphyrin ring or the macromolecules' 3D structure based on the models obtained as in (Table 3 and 4).
Porphyrin rings attached differently for both of normal and sickle $\beta$-globin. Additionally the porphyrin ring orientation is different.

Red bilin against normal and sickle $\beta$-globin show nearly full surface fitting. $21 \mathrm{H}$-Bilin-1(22H)-one against normal $\beta$-globin alone show good surface fitting but not in case of sickle $\beta$-globin.

$21 \mathrm{H}$-Bilin-1(24H)-one fitting is totally different in case of normal and sickle globin.1H-Bilin 1 one show the same fitting profile if the different between the normal and the sickle $\beta$-globin is considered. $22 \mathrm{H}$-Biline (21H-Bilin) is fit partially to normal $\beta$-globin but not the case in the sickle $\beta$-globin.

\section{From the models and the results obtained from this study, some facts can be highlighted:}

1. Molecular docking is a sensitive process. Docking single protein existed in tetrameric form give different result from that if it studied in its monomeric form.

2. The quaternary structure of the macromolecules such as the hemoglobin is very important where any change or reduce in the number of the protein unit will change its 3D conformation hence change the overall ability to bind to its specific molecules such as in case of hemoglobin and porphyrin ring.

3. Molecules could compete each other if they have similar binding sites such as in case of porphyrin ring and the bilins.

4. Big molecules tend to attach to the surface such as in the porphyrin ring and the big bilin.

5. Small molecules tend to penetrate the protein 3D structure such as in case of small bilin and even so they still sensitive to those molecules bind on the protein surface.

6. The in Silico or in computer modeling could find many useful information however in vitro experiment should be the final judgment. Where the molecules under investigation might affect other macromolecules. And in vivo conditions must be some how different and must be conducted for better evaluation.

7. Natural products must take more interest while they are product prove to be safe, hence they are chemically harmless at least if used in the correct amount and dosage.

8. One amino acid change causes such disease which proves the importance of the protein structure/function/specificity.

9. Avoiding such illness condition can be by avoiding the marriage from the same group or the marriage from individuals who have defected trite.

Such avoidance will lead finally to the disappear of such disease after correct generation.

Our understanding to each condition could effect on our macromolecules will let us to normalize the line between our hope and our bodies for the better of our macromolecules. 


\section{Conclusion}

A. platensis could support the patients' with SCA from different points where it's well known for their antiviral, antisickling, vitamin rich, antioxidants, high protein content etc. There is an increasing interest for using $A$. platensis in the SCA research. This study suggests a role for five bilins of the Arthospira. in treating the SCA. For that the requested molecules have been obtained and generated using different software. Six macro molecules and five bilins have been evaluated best on the interaction between each bilin plus he porphyrin ring and each macromolecules. Two bilins show competition with the docked porphyrin ring while the other three bilins did not with correct interaction with the $\beta$-globin 3D structures. It is suggested that some bilins might be used as drugs for treating SCA. However this study did not include any of the in vivo study and such study must be done for more perfect judgment. Additionally, the study includes discussions about the conditions, which might affect on our healthy or modified macromolecules which evaluated in this study and represented by the $\beta$-globin molecules. As antisickling agent bilins will protect the liver from the liver injury due to the effect of the sickle RBCs which will be indirect way to prevent different diabetic diseases including the viruses' infection, fibrosis, etc.

\section{Reference}

1. Smith CA ,Wood EJ. Biological molecule. Springer Netherlands 1991;1-205.

2. Amara AA. The inevitability of balanced lives: genes - foods- Actioninteractions. 2013.

3. Weatherall DJ, Clegg JB, Higgs DR, Wood WG. The hemoglobinopathies In: Scriver CR, Beaudet AL, Sly WS, Valle D (eds). The metabolic basis of inherited disease, 6th ed, MaGraw-Hill:New York;1989.

4. Amara AA. The Need for Early Detection of the Prototype Mutants: Sickle Cell Anemia as a Case Study. doi:104172/jpbS8-006

5. Thompson MW, McInnes RR, Willard HF. Genetics in medicine. Chapter 11: The hemoglobinopathies: Models of molecular disease, $\mathrm{W}$ B Saunders Company,5th ed,1991:247-270.

6. Koskinas J, Manesis EK, Zacharakis GH, Galiatsatos N, Sevastos $\mathrm{N}$, Archimandritis AJ. Liver involvement in acute vaso-occlusive crisis of sickle cell disease: prevalence and predisposing factors. Scand J Gastroenterol. 2007;42(4):499-507. doi: 10.1080/00365520600988212

7. Bauer TW, Moore GW, Hutchins GM. The liver in sickle cell disease: a clinicopathologic study of 70 patients. Am J Med. 1980; 69(6):833837.

8. Mengel CE, Schauble JF, Hammond CB. Infarct-necrosis of the liver in a patient with S-A hemoglobin. Arch Intern Med. 1963; 111(1):93-98. doi:10.1001/archinte.1963.03620250097013

9. Fishbone G, Nunez D Jr, Leon R, Paz G, Isturiz P, McLoughlin C. Massive splenic infarction in sickle cell-hemoglobin $C$ disease: angiographic findings. AJR Am J Roentgenol. 1977;129(5):927-928. doi: 10.2214/

\section{ajr.129.5.927}

10. Schubert TT. Hepatobiliary system in sickle cell disease. Gastroenterology. 1986;90(6):2013-2021.

11. Rosenblate HJ, Eisenstein R, Holmes AW. The liver in sickle cell anemia: a clinical-pathologic study. Arch Pathol. 1970;90(3):235-245.

12. Ebert EC, Nagar M, Hagspiel KD. Gastrointestinal and Hepatic Complications of Sickle Cell Disease. Clin Gastroenterol Hepatol. 2010; 8(6):483-489. doi: 10.1016/j.cgh.2010.02.016

13. PubChem. PubChem open Chemistry Database; NIH; US National library of Medicine; National Venter for Biotechnology information.

14. Blast Madden T. The NCBI Handbook. The BLAST Sequence Analysis Tool. 2002; McEntyre J, Ostell J, Interne Bethesda MD: National Center for Biotechnology Information US.

15. Shaanan B. 1HHOPDB (Structure of human oxyhaemoglobin at 21 angstroms resolution) Protein Data Bank in Europe EMBL-EBI. J. Mol. Biol. 1983; 31-59.

16. Sali A, Blundell TL. Comparative protein structure modeling by satisfaction of spatial restraints. J Mol Biol. 1993;234(3):779-815.

17. Ritchie DW. Evaluation of Protein Docking Predictions Using Hex 3.1 in CAPRI Rounds 1 and 2. proteins. 2003;52(1):98-106. doi: 10.1002/ prot.10379

18. Ritchie DW, Kemp GJ. Protein Docking Using Spherical Polar Fourier Correlations. PROTEINS. 2000;39(2):178-194.

19. Ritchie DW, Kemp GJL. Fast Computation, Rotation, and Comparison of Low Resolution Spherical Harmonic Molecular Surfaces. J Comp Chem. 1999;20:383-395.

20. Ritchie DW. Evaluation of Protein Docking Predictions Using Hex 3.1 in CAPRI Rounds 1 and 2. Proteins. 2003;52(1):98-106. doi: 10.1002/ prot.10379

21. Discovery Studio Visualizer V41014169. 2005-2014; Accelrys Software Inc.

22. Kapff CT, Jandl JH. Blood, atlas and sourcebook of hematology. 1st ed. Little Brown and Company Boston USA. 1981;56-57.

23. Aguileta G, Bielawski JP, Yang Z. Evolutionary rate variation among vertebrate beta globins genes: implications for dating gene family duplication events. Gene. 2006;380(1):21-29. doi: 10.1016/j. gene.2006.04.019

24. Kumar R, Sagar C, Sharma D, Kishor P. $\beta$-globin genes: mutation hotspots in the global thalassemia belt. Hemoglobin. 2015; 39(1):1-8. doi: 10.3109/03630269.2014.985831

25. Livingstone FB. Simulation of the diffusion of the beta-globin variants in the Old World. Hum Biol. 1989;61(3):297-309.

26. Sharaf M, Amara A, Aboul-Enein A, Helmi S, Ballot A, Astani A, et al. Molecular authentication and characterization of the antiherpetic activity of the cyanobacteriumA. fusiformis. Pharmazie. 2010;65(2):132-136.

27. Amara AA, Steinbüchel A. New Medium for Pharmaceutical Grade Arthospira International Journal of Bacteriology 2013;2013:9. doi: $101155 / 2013 / 203432$ 